\title{
All-fiber ultrafast image detection enabled by deep learning
}

\section{Zhoutian Liu}

Tsinghua University

\section{Lele Wang}

Tsinghua University

\section{Yuan Meng}

Tsinghua University

\section{Tiantian He}

Tsinghua University

\section{Sifeng He}

Tsinghua University

\section{Yousi Yang}

Tsinghua University

\section{Liuyue Wang}

Tsinghua University

Jiading Tian

Tsinghua University https://orcid.org/0000-0002-6764-1086

\section{Dan Li}

Tsinghua University

\section{Ping Yan}

Tsinghua University https://orcid.org/0000-0002-8177-7019

\section{Mali Gong}

Tsinghua University

Qiang Liu

Tsinghua University

Qirong Xiao ( $\sim$ xiaoqirong@mail.tsinghua.edu.cn )

Tsinghua University https://orcid.org/0000-0001-9419-3939

\section{Article}

Keywords: image detection, deep learning, engineering, chemistry, material science, biomedicine 
DOl: https://doi.org/10.21203/rs.3.rs-568362/v1

License: (c) (1) This work is licensed under a Creative Commons Attribution 4.0 International License. Read Full License

Version of Record: A version of this preprint was published at Nature Communications on March 17th, 2022. See the published version at https://doi.org/10.1038/s41467-022-29178-8. 


\begin{abstract}
Detection of dynamical scenes at ultrafast speeds serves as a foundation for modern engineering, chemistry, material science and biomedicine, et al. For biomedical applications, in vivo microscopic imaging is often required, which has led to the development of fiber-probe-based endoscopy. However, the combination of ultrafast image acquiring with fiber endoscopy has not yet been achieved, which is vital for exploration of transient biomedical phenomena. Here, we propose a scheme of all-fiber image detection at an ultrafast speed without any free-space optical elements. Image detection is achieved based on the transformation of two-dimensional spatial information into one-dimensional temporal pulsed signal streams by leveraging the high intermodal dispersion in a multimode fiber. Deep learning algorithms are subsequently deployed to reconstruct the images detected by the fiber probe from the temporal waveforms acquired at the other end of the fiber. The fiber probe can directly detect micron-scale objects without any bulk objective, and image detection has been experimentally realized with a high frame rate (15 Mfps), a large frame depth $\left(10^{4}\right)$ and extremely short shutter time ( $30 \mathrm{ps}$ ) simultaneously. This ultrafast detection scheme, combined with high mechanical flexibility and high level of integration, can stimulate future research on exploring various in-vivo ultrafast phenomena.
\end{abstract}

\title{
Introduction
}

Ultrafast image detection is vital for observing microscopic and transient physical phenomena ${ }^{1}$. To date, silicon-based imaging sensors, charge-coupled device (CCD) or complementary metal-oxidesemiconductor (CMOS) cameras, have achieved imaging speeds of up to millions of frames per second (fps) ${ }^{2}$. Some advanced systems are also invented for even faster transient imaging, reaching trillions of fps, including sequentially timed all-optical mapping photography (STAMP) ${ }^{3}$, frequency-domain tomography ${ }^{4}$, femtosecond time-resolved optical polarimetry ${ }^{5}$, and compressed ultrafast spectral photography ${ }^{6}$. These advanced technologies have helped researchers better understand various transient phenomena, such as lattice dynamics ${ }^{7}$, hot-electron diffusion ${ }^{8}$, evolution of laser ablation ${ }^{9}$ and production of electronic plasmas ${ }^{10}$. However, in some other fields, especially in vivo applications ${ }^{11}$, ultrafast detection requires new technologies for imaging in such narrow spaces, for which emerging fiber-based detection technology has unique advantages.

In contrast to bulk imaging systems, fiber-based imaging systems feature high mechanical flexibility, compact sizes and resistance to ambient interference. These features have made fiber-based imaging a competitive candidate for detecting images under special circumstances, for example, in environments with high temperatures, pressures or radiation levels. Fiber probes can also penetrate deep into narrow spaces for endoscopy, which is essential in fields such as biomedicine ${ }^{12}$ and microfluidics ${ }^{13}$. Fiber endoscopy with a high frame rate is especially necessary in some special scenarios. For instance, a fiber probe can be inserted into the cerebral cortex to examine the fast signals of neural activation ${ }^{14}$ or used in vivo to observe chemical dynamics in living tissues ${ }^{15}$. In physics and engineering, such probes can also be used for observing ultrafast physical reactions in closed containers ${ }^{16}$ or exploring the fuel injection dynamics in internal combustion engines.

However, for currently prevalent fiber-based imaging systems, their ability to ultrafast detection is 
limited by the CCD or CMOS cameras that serve as essential parts in their systems. The basic principle of these technologies is to analyze the light fields at the output fiber facet and reconstruct the $2 \mathrm{D}$ images using such as transmission matrix methods and deep learning methods ${ }^{14,17}$. Due to such a principle, they must detect the fields of different frames at a fixed position, which means that they can only use conventional single-sensor cameras (special cameras such as rotating-mirror cameras ${ }^{18}$ and framing cameras that have higher frame rates are inapplicable). While the single imaging sensor faces the challenge of slow readout process from the pixel arrays to the memory ${ }^{19}$, both the frame rate, frame depth (number of frames that can be captured in a single shot) and shutter time (exposure time of one frame) of those fiber-based imaging systems are limited. To the best of our knowledge, the world's fastest single-sensor camera has a frame rate of $10 \mathrm{Mfps}$, a frame depth of 256 frames and shutter time of $50 \mathrm{~ns}$ (see https://www.shimadzu.com/an/products/materialstesting/high-speed-video-camera/hyper-vision-hpv-x2/index.html), which puts an upper limit on these systems. In addition, free-space optical elements are also commonly needed in collection of the output fields, which reduce the level of integration and make those systems susceptible to environmental disturbances.

Fortunately, a time-stretching imaging method (or serial time-encoded amplified microscopy, abbreviated as STEAM) $)^{20-22}$ has been proposed to eliminate these pixelated sensors by encoding the spatial information of objects into time-domain signals, which requires only a one-pixel detector. Since each optical pulse can carry the information of one image frame, a high frame rate can be achieved by recording the temporal signals of a pulse train with a high repetition rate. However, such systems require bulk spatial dispersers, which are not compatible with the fiber endoscopy.

To combine the advantages of the time-stretching method and the fiber endoscopy, we propose a novel method to enable all-fiber ultrafast detection of images. Using a single multimode fiber (MMF) as the probe, real-time image acquisition with a frame rate of over $15 \mathrm{Mfps}$ and a shutter time of approximately $30 \mathrm{ps}$ is experimentally demonstrated, in which 10,000 frames can be recorded in a single shot. Leveraging the intermodal dispersion effect in an MMF, we transform two-dimensional (2D) spatial information into one-dimensional (1D) time-domain pulsed waveforms. A U-Net neural network model is trained to reconstruct the images from the temporal waveforms recorded by an ultrafast photodiode connected to the output end of the fiber. In addition, we propose an all-fiber structure for the first time by combining a fiber-output pulse laser, a triple-cladding fiber probe and a side-pump coupler. Such a scheme enables unprecedentedly high levels of integration and system stability.

\section{Results}

Principles. The light fields in an MMF can be resolved into a set of orthogonal spatial modes ${ }^{23}$ that enable the transmission of spatial information. It has been verified that the information of images with $4 N$ resolvable features can be carried in a single MMF, where $N$ is the number of spatial modes per polarization ${ }^{24}$. When light scattered by an object is collected by an MMF, various fiber modes will be excited to different degrees. When we use an ultrafast pulse laser as the illumination source, the energy of each pulse entering the MMF can be dispersed into different modes. Because the different modes have different group velocities, the pulses in these modes will arrive at the other end of the MMF with different time delays. If the intermodal dispersion of the MMF is sufficiently 
large, after transmission through the MMF, a pulse with a temporal duration of less than the delay difference between two modes will be split into a number of isolated subpulses in the time domain, as schematically shown in Fig. 1. If the power of the pulse is sufficiently low and its wavelength bandwidth is sufficiently narrow, both the chromatic dispersion and nonlinear effects in the MMF can be ignored, resulting in the pulse evolution being dominated by the intermodal dispersion ${ }^{25,26}$ (see Supplementary Note 1 for details). Therefore, the temporal distribution of the train of subpulses will depend on the mode composition of the original pulse, which is determined by the spatial distribution of the object. Hence, the spatial information of objects can be encoded into the time waveforms of the output pulses.

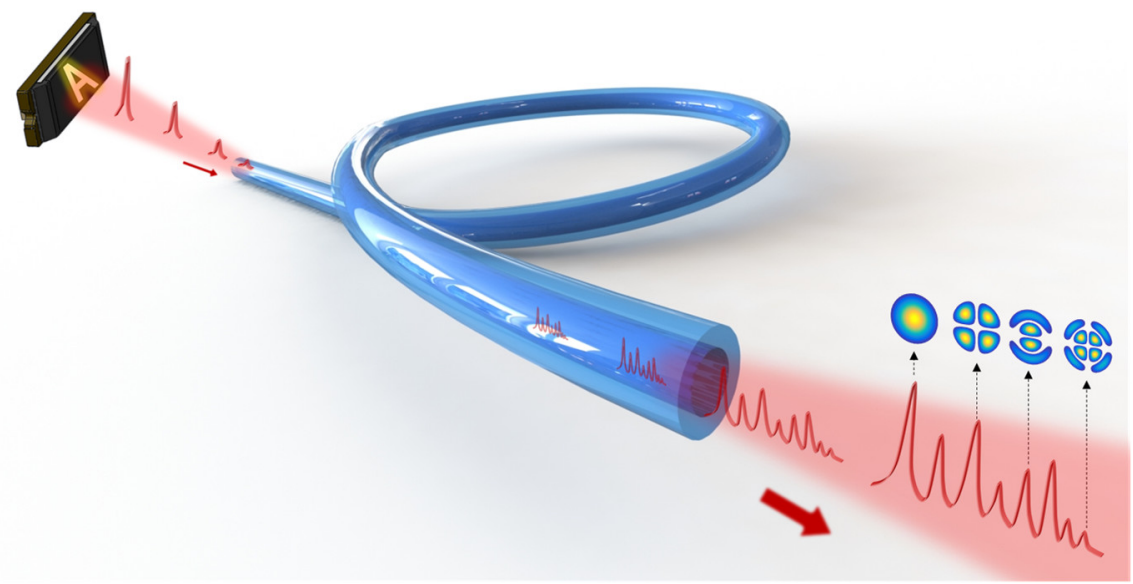

Fig. 1 The evolution of ultrashort pulses in a long MMF with large intermodal dispersion.

Experimental setup. The structure of the system is illustrated in Fig. 2(a). The illumination pulses from a mode-locked fiber laser are directly coupled into the fiber probe by a side-pump coupler ${ }^{27}$. After approximately 2 meters of transmission, the illumination pulses emerge from the fiber probe to illuminate the intensity patterns displayed by a digital micromirror device (DMD). Then, the light reflected from the patterns reenters the fiber probe, as shown in Fig. 2(c). Thus, the illumination and reception of light are integrated into a single fiber probe. The other end of the fiber probe is spliced with a $1-\mathrm{km}$ MMF $(50 / 125 \mu \mathrm{m}$ and numerical aperture $(\mathrm{NA})=0.22)$, in which the spatial information carried in the signal pulses is transformed into temporal waveforms. This length guarantees that the delay differences between different modes are sufficiently large to cause each a signal pulse to split into a burst of subpulses. The temporal waveforms of the pulses at the other end of the MMF are detected by an ultrafast photodetector and stored into the memory of an oscilloscope instantly. In the training stage, different displayed images and the corresponding waveforms are used to train the neural network model. After training, the network is capable of recovering new images directly from the acquired waveforms, as shown in Fig. 2(b).

The fiber probe is a triple-cladding fiber, and both the core and the second cladding layer can transmit light. The structure of the side-pump coupler, where the illumination light is coupled into the second cladding layer of the fiber probe (see Supplementary Note 2 for the detailed structure), is schematically shown in Fig. 2(d). Although the light reflected by the DMD will enter both the core and cladding of the fiber probe, only the light in the core (signal light) can enter the MMF due to the NA and diameter match between the fiber probe core and the MMF core. The end of the fiber probe is fused into a microball with a $580 \mu \mathrm{m}$ diameter, as shown in Fig. 2(e), which serves to 
produce more uniform and focused illumination (in the absence of this microball, the beam emerging from the cladding of the fiber would have an annular shape). This probe can be directly moved very close to microscale objects for imaging, with no requirement of objectives that are vital for conventional cameras. To demonstrate this, the fiber-end ball probe was placed very close to the surface of the DMD, such that it could receive only light returning from a very small part of the DMD. This small region was measured to have an area of approximately $200 \times 200 \mu^{2}$, in which images of approximately $28 \times 28$ pixels can be displayed.

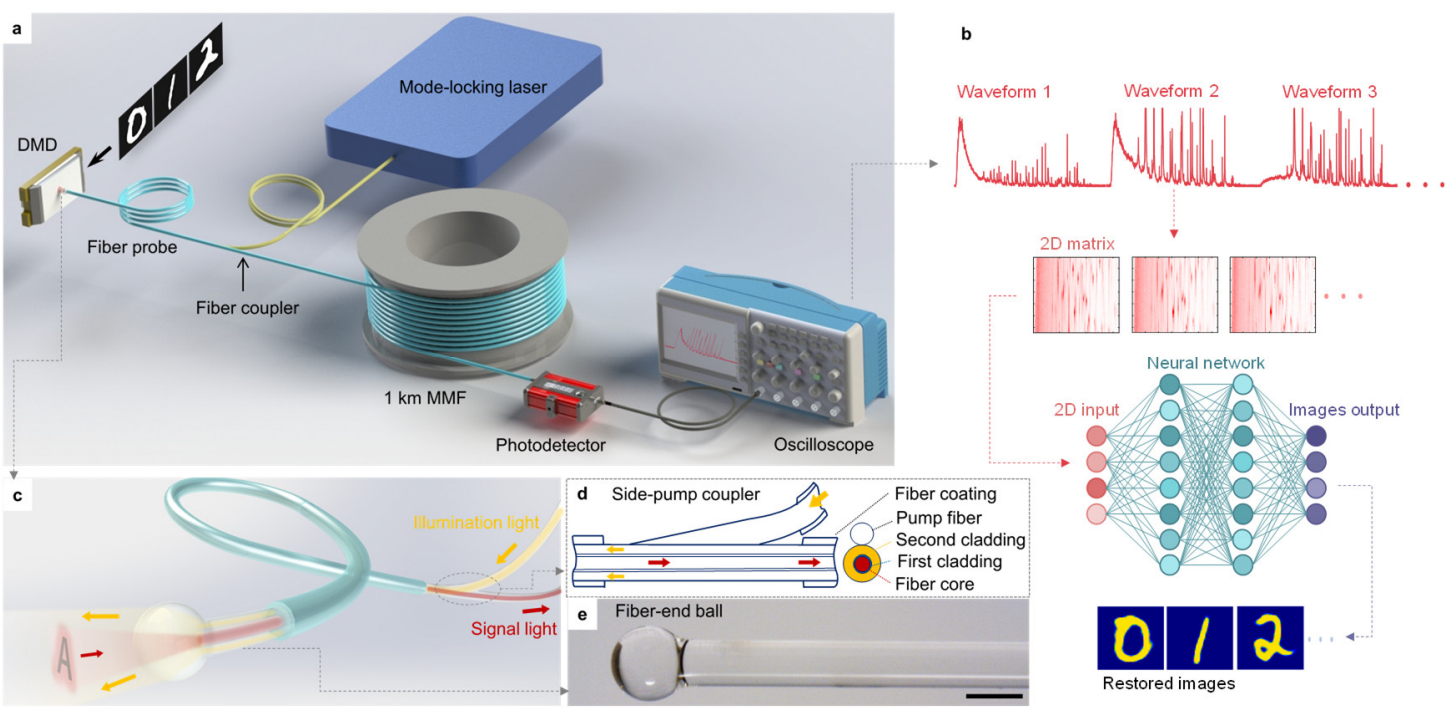

Fig. 2 (a) Schematic of the experimental setup. (b) Flow of the reconstruction process from waveforms to images. (c) Schematic of the fiber-end ball and the side-pump fiber coupler and the flow of illumination light and signal light.

(d) Structure of the side-pump coupler and the triple-cladding fiber probe, where the illumination light is coupled into and transmitted in the cladding of the fiber, while the signal light is collected and transmitted in the fiber core in the opposite direction. (e) A micrograph of the fiber-end ball. Scale bar: $500 \mu \mathrm{m}$.

Image recovery. Fig. 3(a) and 3(b) show several example images from the MNIST dataset ${ }^{28}$ and their corresponding temporal waveforms. We see that after transmission through the long MMF, a 27.5-ps input pulse splits into a burst of subpulses over approximately $45 \mathrm{~ns}$ (see Supplementary Note 3 for more waveform details). A U-Net model was trained on 19000 waveform/image pairs to learn the mapping (the training details are described in the Methods section). Using the trained model, we could directly recover other new images from the corresponding acquired waveforms. The recovery results corresponding to these example images are shown in Fig. 3(c). The results for 1000 test images show an average fidelity (calculated as the 2D correlation) of $81.8 \%$ and average structural similarity index measure (SSIM, which correlates well with human perception) of 0.78 . Compared with previous fiber endoscopy technologies, which generally operate at low frame $\operatorname{rates}^{17,29,30}$, our scheme shows comparable performance in terms of image quality. 


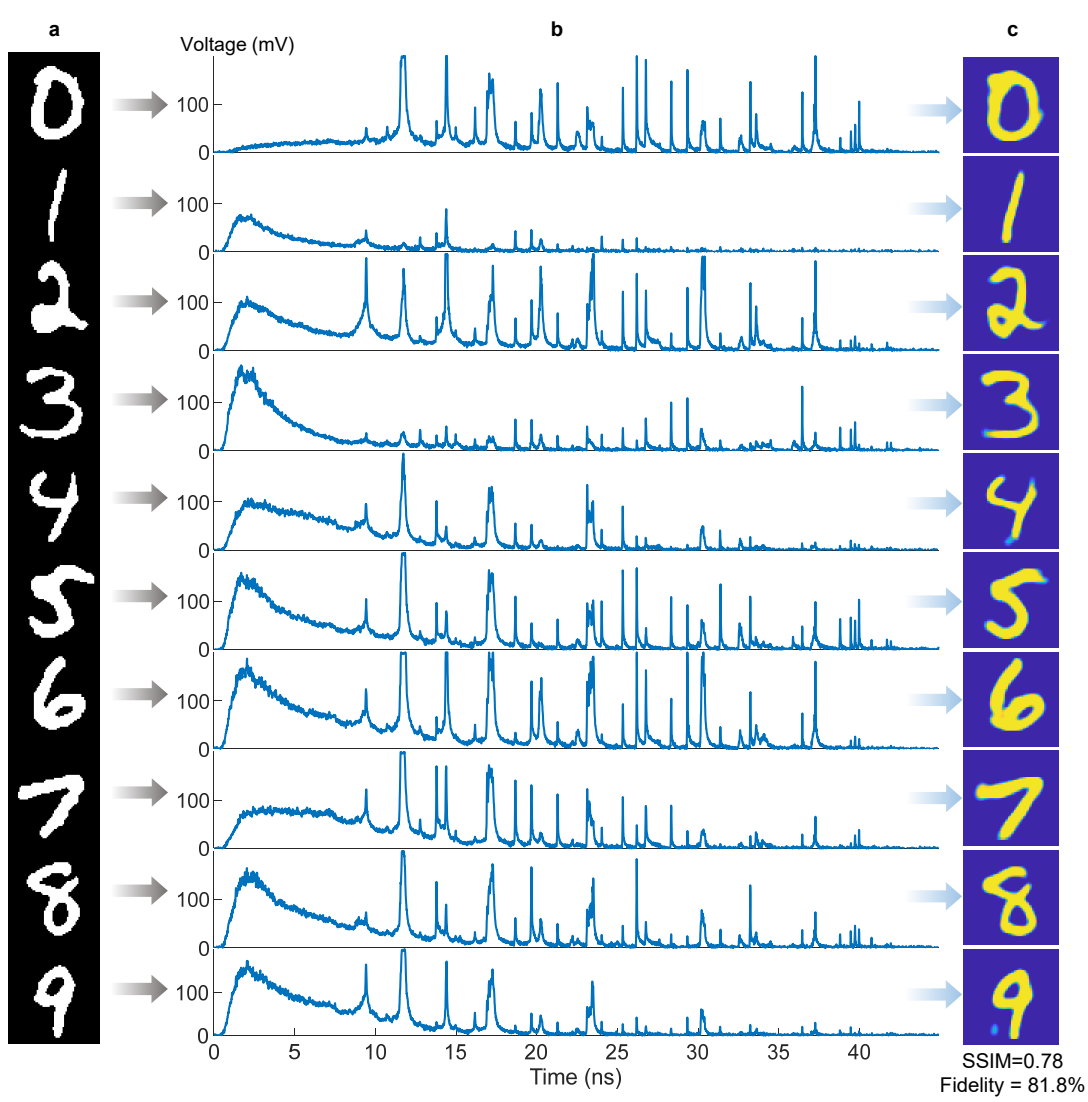

Fig. 3 (a) Example images of digits $0-9$ selected from the test database and (b) their corresponding output waveforms. (c) Images recovered from the waveforms.

We also tested the reconstruction performance for several different types of images, including handwritten letters from the EMNIST dataset ${ }^{31}$ and patterns of clothes from the Fashion-MNIST dataset $^{32}$. After a similar training process, the images could be recovered, and some examples are shown in Fig. 4, along with the average fidelity and SSIM. The results show the high practicability of our scheme. While the waveform corresponding to one pulse corresponds to one image, successive pulses can enable the detection of images at a frame rate of $15 \mathrm{Mfps}$, consistent with the repetition rate of the pulse source. Moreover, the shutter time is only approximately $30 \mathrm{ps}$, which is equal to the time duration of one pulse.

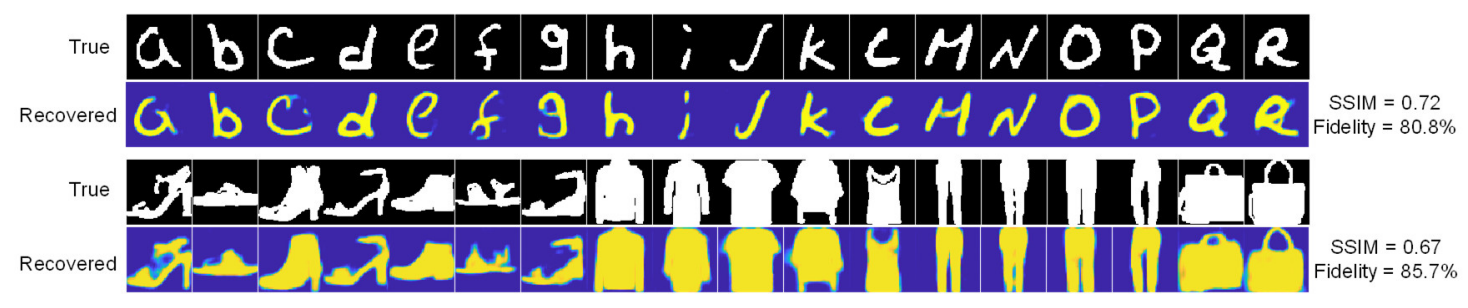

Fig. 4 Some example images of letters and clothes and the retrieved images.

Finally, we verified that instead of utilizing the waveforms with the highest sampling rate $\left(10 \mathrm{ps}^{-1}\right)$ recorded by the oscilloscope, when the waveforms are down sampled to a small number of feature points, the images can still be recovered at the cost of a slight reduction in imaging quality (see 
Supplementary Note 5). We down sampled the waveforms to only 256 points, which were then fed to the network. The results for the test images of handwritten digits show an average fidelity and SSIM of $77.2 \%$ and 0.73 , respectively. These findings indicate that the main information is encoded only in certain feature points of the waveforms. This property can help to reduce the requirements of the sampling circuits and improve the signal processing speed in real applications.

Image classification. This system can also be used for high-speed classification, which has great value in fields such as biomedicine and microfluidics ${ }^{13}$. Here, we demonstrate the classification of handwritten digits based on the acquired waveforms. We use a single convolutional neural network $(\mathrm{CNN})$ to learn the classification while either the retrieved images output from the U-Net or the original waveforms data are used as the input of network (see Supplementary Note 4 for details). In testing on 1000 new images, a high accuracy of $91.5 \%$ was achieved as indicated by Fig. 5, indicating that the combination of the U-Net and CNN can exhibit a higher accuracy. We note that image detection through such long fibers has been a major challenge for conventional multimode imaging systems $\mathrm{s}^{30}$ because the disturbance grows more severe as the fiber length increases ${ }^{29}$, making the recovery more difficult (the accuracy dropped to less than $70 \%$ for a $1-\mathrm{km}$ MMF in a previous research). However, in our scheme, the classification accuracy remains at such a high level under the same length, showing high interference immunity and practicability. This superiority can probably be attributed to low crosstalk between different modes, when the pulse energy in them has been separated after transmission over a certain distance in the MMF, thus, the energy coupling between different modes is suppressed. Such a feature makes our scheme also well suitable for longdistance detection.
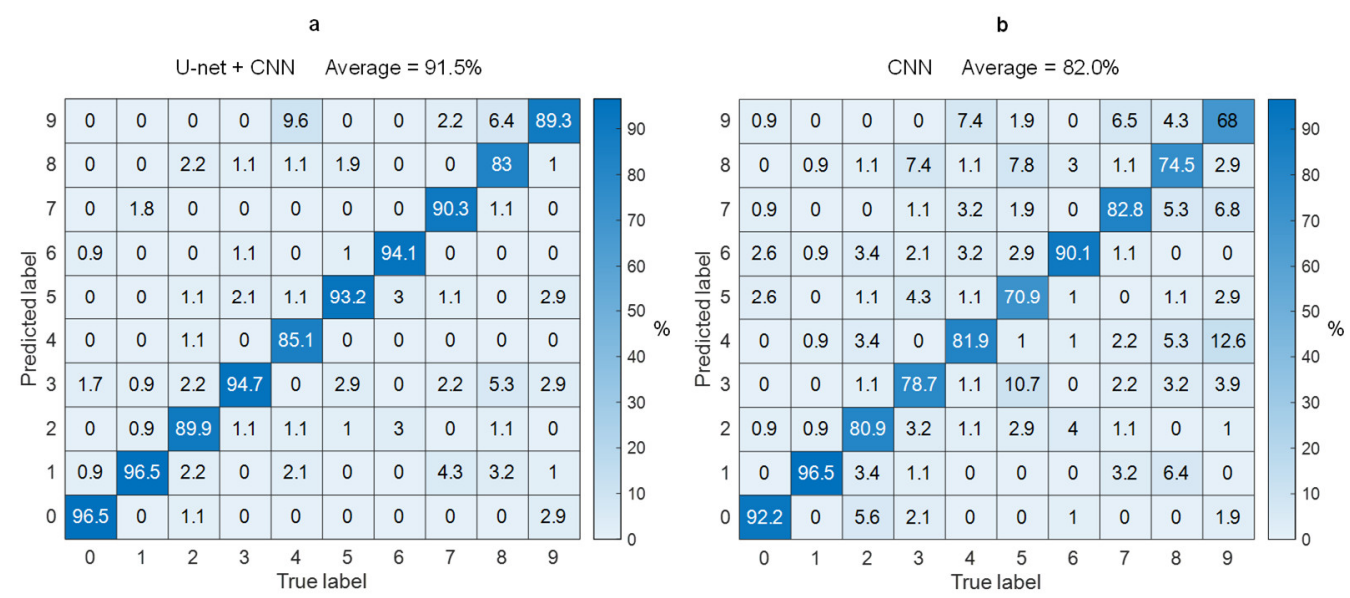

Fig. 5 Confusion matrixes for the classification of 1000 test images using (a) a combined U-Net and CNN model and (b) the CNN alone. These matrixes present the statistics of what proportion of each digit is correctly classified. Each matrix element gives the possibility of instances in which one number (true label) is recognized as another number (predicted label).

Ultrafast detection. To verify the feasibility of ultrafast detection, we adjusted the time scale of the oscilloscope to the maximum ( $650 \mu \mathrm{s})$ so that it would store approximately 10,000 waveforms in a single record. Although the highest refresh rate of the DMD used here is limited to $4.3 \mathrm{kHz}$, preventing it from displaying an ultrafast video that matches our detection frame rate of $15 \mathrm{Mfps}$, 
the refresh processes when the DMD switches from one image to another are nearly transient and occurre within $3 \mu$ s (see the recorded waveforms below). Thus, we chose to detect such a refresh process using our system to reveal the detailed refresh process in such a short time. We set the DMD to periodically display two images and simultaneously record the time signals, as shown in Fig. 6(b). The detailed waveforms corresponding to one refresh process (marked with a red circle) are shown in Fig. 6(a), where we can see the process of the waveform corresponding to an image "3" gradually changing to the waveform corresponding to an image " 0 " within $3 \mu \mathrm{s}$. The retrieved successive frames are shown in the insets (a1 - a17), from which we can understand the refresh process of the DMD. The whole refresh process can be divided into 3 stages. In stage 1 (insets a1 - a5), the DMD initially displays the " 3 " image, which means that the micromirrors in regions (i) and (ii) of the DMD (see Fig. 6e) are in the "on" state, while the others are in the "off" state. The states of the micromirrors are explained in Fig. 6(d), where region (ii) represents the overlap between the " 0 " and " 3 " images. When the DMD starts to refresh to " 0 " image, the micromirrors in regions (i) and (iii) rotate to the opposite directions ${ }^{33}$, causing the light in region (i) to fade away. In stage 2 (insets a6 - a14), only the light from region (ii) can be observed because the micromirrors there will hold the states of "on". In stage 3 (insets a15 - a17), the light from region (iii) appears, indicating that the corresponding micromirrors have rotated into the "on" state. Thus, the image " 3 " has been refreshed to the image " 0 ". For comparison, we also used a high-speed camera to record the refresh process (see the Methods section for details), and the real images captured are shown in Fig. 6(c). We can see that the change in the patterns is consistent with what we have observed using our system.

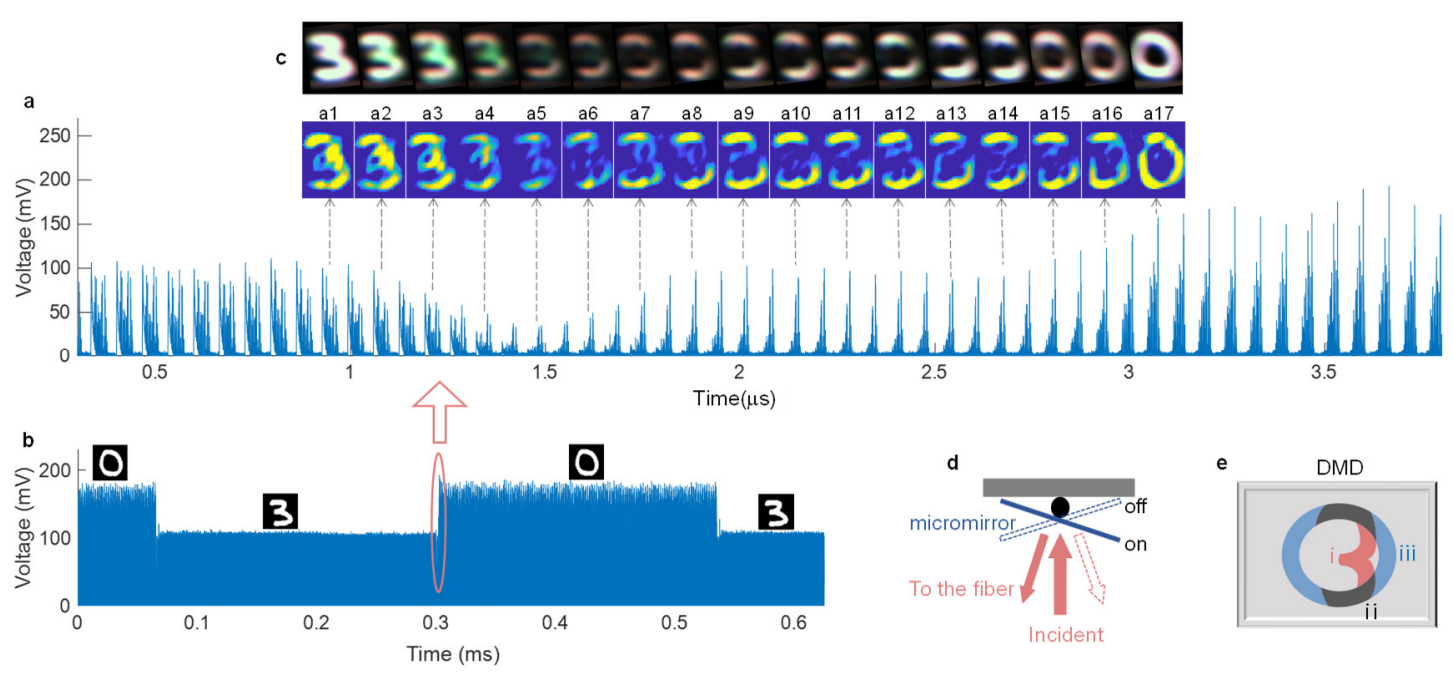

Fig. 6 (a) The waveforms collected during the transient time when the DMD refreshes from an image " 3 " to an image "0". Insets (a1 - a17) show the images reconstructed from some of these waveforms. (b) The waveforms recorded with the DMD periodically displaying two images, from which we see that each image has an exposure time of 235 $\mu$ s, which is spent by the DMD in loading the data for the next image into the memory cells beneath its pixels. After all the data have been loaded, the DMD switches to the other image within only $3 \mu \mathrm{s}$, as marked by the red circle. (c) The real images of the DMD captured during the refresh process using a high-speed camera. (d) Structure of one pixel of the DMD. A DMD chip has many micromirrors on its surface, which correspond to the pixels in the image to be displayed. These micromirrors can be individually rotated by approximately $\pm 12^{\circ}$ to an "on" or "off" state. In the "on" state, the illumination light is reflected back to the fiber probe. In the "off" state, the light is directed in another direction. Thus, the array of micromirrors can produce intensity modulations on the light field. When the 
DMD refreshes its current image, all micromirrors move to their assigned states at the same moment. (e) Schematic of the patterns of the digits " 3 " and " 0 " displayed on the DMD chip. When the DMD switches from " 3 " to " 0 ", the micromirrors in the overlapping region of the two images (region ii) do not change their states, while those in regions (i) and (iii) rotate to the opposite directions.

\section{Discussion}

Because the proposed scheme needs only a single photodiode rather than pixelated sensors, it can be easily applied to other wavelengths. For example, considering that the InGaAs-based photodiode used here has a high sensitivity over a broad wavelength band from $1 \mu \mathrm{m}$ to $1.6 \mu \mathrm{m}^{34}$, while the silica fiber has very low attenuation in this band, our scheme can be easily extended to other wavelengths in this band. This will be of great help in real applications because conventional Sibased CCD and CMOS cameras are sensitive only to wavelengths below $1.1 \mu \mathrm{m}^{19}$. Our method also has the potential to operate in the mid-infrared and $\mathrm{THz}$ bands considering the maturity of photodetectors in these bands. In addition, in the mid-infrared, the use of a fluoride-glass fiber can significantly reduce the optical loss, while in the $\mathrm{THz}$ band, much work has focused on the development of waveguides with reduced loss and dispersion ${ }^{35}$, which make it possible to develop long waveguides with high intermodal dispersion in these bands. Thus, we may apply the same principle to these bands. This will be helpful for detecting certain materials that have a strong response only at these wavelengths or for detection under special conditions in which only light in these bands can be transmitted with low loss. Summing up the above, our scheme can offer a new approach for observing vivid physical phenomena in a vast number of scenarios.

The performance of our demonstrated proof-of-principle system may be further improved. The wavelength of the source used here $(1064 \mathrm{~nm})$ is much longer than those adopted in most previous studies $^{17,23,29,30}$, which will result in a much smaller number of excited modes and, thus, much less spatial information carried in the MMF. Hence, by using an MMF with a larger core and higher NA, more spatial information can be collected, and the resolution of the images that can be recovered will, in theory, be much higher. In addition, the shutter time can be made even shorter to enable the detection of faster events by using shorter pulses. More importantly, if one splices a fiber amplifier to the end of the MMF, the pulse signals can be significantly amplified, which will greatly enhance the sensitivity of the detection system to make it suitable for detecting very weak signals. Moreover, because the illumination zone and intensity are limited for this fiber probe, the current system is suitable only for detecting small objects. If one wishes to detect a larger object, an objective can be used in front of the fiber probe to couple more light from the object into the probe. Additionally, if one wishes to use a brighter illumination, an auxiliary illumination can be adopted at the cost of reducing the systems' level of integration, as discussed in Supplementary Note 6.

Our scheme can be further modified to detect 3D objects by combining it with the existing time-offlight technique ${ }^{36-38}$, in which ultrafast pulses are generally applied to illuminate the objects of interest and an ultrafast camera is used to detect the reflected light at different arrival times. Because light reflected from different depths on the object will arrive at the camera with different time delays, the variations in the $2 \mathrm{D}$ images captured over time can reveal the $3 \mathrm{D}$ information of the object. The system we present here is naturally compatible with the time-of-flight method because we also adopt an ultrafast pulse laser for illumination. If we use the fiber probe to detect a 3D object, the temporal 
waveforms will contain both depth information and 2D spatial information. Thus, through specific reconstruction algorithms, it will be possible to recover the $3 \mathrm{D}$ information encoded in these ultrafast time signals.

\section{Methods}

Experiments. The laser source is a homemade Yb-doped mode-locked fiber laser with a spectral width of $0.2 \mathrm{~nm}$. The pulse power is carefully set to strike a balance between strong signals that can be detected by the photodetector and low nonlinear effect suffered by the pulses in the MMF. The large mode area of the 1-km MMF can also help to suppress nonlinear effects ${ }^{39}$. The step-index core of the MMF can provide much larger intermodal dispersion than a graded-index core ${ }^{40}$. The fiber probe is a triple-cladding fiber with dimensions of 50/70/360 $\mu \mathrm{m}$ (NUFERN FUD-4658, BDS50/70/360-22FA-HP). The NAs of the core and the second cladding layer of the triple-cladding fiber are 0.2 and 0.46 , respectively. The core of the fiber probe approximately matches that of the MMF. The homemade fiber coupler couples light from the source into the second cladding layer of the probe. The fiber-end ball at the end of the probe was made via fusion with a fusion splicer (Fujikura FSM-100P+). Although we adopt a pulse laser, the DMD can perform only grayscale modulation of continuous light, so that all images from the datasets must be binarized before being loaded into the DMD.

The high-speed camera (MotionBLITZ EoSens ${ }^{\circledR}$ mini) used in the ultrafast experiment has a frame rate of $40 \mathrm{kHz}$, which is too slow to record the refresh process of the DMD in real time. Thus, the images showing this transient process in Fig. 6(c) were not actually captured during a single refresh process. Instead, they were obtained using the following method. First, we acquired a large number of images while the DMD was periodically switching between the two display images. Because a single refresh process occupies only a very small proportion of a switching period, as shown in Fig. 6(b), only a small number of images were captured exactly during the refresh processes. Because these images tended to record different states of the process, they could be combined to represent a continuous refresh process. The exposure time of the camera was set to the minimum to capture these transient states.

Training. The original images were interpolated into $64 \times 64$ matrixes as the output, while the 4096point waveforms were reshaped into $64 \times 64$ matrixes as the input to the network. In the data collection stage, for each type of image, 20000 different images of handwritten digits were successively displayed on the DMD, and the corresponding output time signals were collected. In the training stage, the data were divided into 17000 waveform/image pairs as the training set, 2000 waveform/image pairs as the validation set and 1000 waveform/image pairs as the test sets. The UNet model converged after approximately 30 epochs of iteration. Then, the 1000 test waveforms were fed to the trained model to reconstruct the corresponding images. The structure of the U-Net network is shown in Supplementary Note 4.

\section{References}

1 Feist, A., Silva, N. R. D., Liang, W., Ropers, C. \& Schfer, S. Spatio-Temporal Probing of Lattice Dynamics in Graphite by Ultrafast TEM. European Microscopy Congress 2016: Proceedings. 330-331 (2016). 


5

El-Desouki et al. CMOS Image Sensors for High Speed Applications. Sensors (2009).

Nakagawa, K. Sequentially timed all-optical mapping photography for observation of ultrafast phenomena. 2015 Opto-Electronics and Communications Conference (OECC). 15650105 (2015).

$4 \quad$ Li, Z., Zgadzaj, R., Wang, X., Chang, Y. Y. \& Downer, M. C. Single-shot tomographic movies of evolving light-velocity objects. Nat. Commun. 5, 3085 (2014).

5 Xiaofang et al. High-frame-rate observation of single femtosecond laser pulse propagation in fused silica using an echelon and optical polarigraphy technique. Appl. Opt. 53, 8395-8399 (2014).

6 Wang, P., Liang, J. \& Wang, L. V. Single-shot ultrafast imaging attaining 70 trillion frames per second. Nat. Commun. 11, 2091 (2020).

7 Feist, A., Silva, N. R. D., Liang, W., Ropers, C. \& Schfer, S. Spatio-Temporal Probing of Lattice Dynamics in Graphite by Ultrafast TEM. European Microscopy Congress 2016: Proceedings. (2016).

Block, A., Liebel, M., Yu, R., Spector, M. \& Hulst, N. F. V. Tracking ultrafast hot-electron diffusion in space and time by ultrafast thermomodulation microscopy. Sci. Adv. 5, eaav8965 (2019).

9 Zyung, T., Kim, H., Postlewaite, J. C. \& Dlott, D. D. Ultrafast imaging of $0.532-\mu \mathrm{m}$ laser ablation of polymers: Time evolution of surface damage and blast wave generation. J. Appl. Phys. 65, 4548-4563 (1989).

Gawelda, W. et al. Ultrafast imaging of transient electronic plasmas produced in conditions of femtosecond waveguide writing in dielectrics. Appl. Phys. Lett. 93, 231115 (2008).

Osmanski, B. F. et al. Ultrafast imaging of blood flow dynamics in the myocardium. IEEE Trans. Med. Imaging 31, 1661-1668 (2012).

Deffieux, T., Gennisson, J.-L., Tanter, M., Fink, M. \& Nordez, A. Ultrafast imaging of in vivo muscle contraction using ultrasound. Appl. Phys. Lett. 89, 184107 (2006). Li, Y. et al. Deep cytometry: deep learning with real-time inference in cell sorting and flow cytometry. Sci. Rep. 9, 11088 (2019).

Mehta, A. D., Jung, J. C., Flusberg, B. A. \& Schnitzer, M. J. Fiber optic in vivo imaging in the mammalian nervous system. Curr. Opin. Neurobiol. 14, 617-628 (2004). cell physiology. Biosystems 83, 217-224 (2006). Dufour, J., Murat, D., Dufour, X. \& Foos, J. Experimental Observation of Nuclear Reactions in Palladium and Uranium-Possible Explanation by Hydrex Mode. Fusion Sci. Technol. 40, 91 106 (2001).

17 Rahmani, B., Loterie, D., Konstantinou, G., Psaltis, D. \& Moser, C. Multimode optical fiber transmission with a deep learning network. Light: Science \& Applications 7, 69 (2018).

Wu, L. et al. Analysis and Design of a CMOS Ultra-High-Speed Burst Mode Imager with InSitu Storage Topology Featuring In-Pixel CDS Amplification. Sensors 18, 3683 (2018). (2009). of fast dynamic phenomena. Nature 458, 1145-1149 (2009).

Karpf, S. et al. Spectro-temporal encoded multiphoton microscopy and fluorescence lifetime 
imaging at kilohertz frame-rates. Nat. Commun. 11, 2026 (2020).

22 Liao, R., Hon, N. K., Buckley, B. W., Diebold, E. D. \& Jalali, B. Chromo-modal dispersion for optical communication and time-stretch spectroscopy. Opt. Lett. 46, 500-503 (2021).

Zhu, C. et al. Image reconstruction through a multimode fiber with a simple neural network architecture. Sci. Rep. 11, 896 (2021).

24 Mahalati, R. N., Gu, R. Y. \& Kahn, J. M. Resolution limits for imaging through multi-mode fiber. Opt. Express 21, 1656-1668 (2013).

25 Lee, J. \& Kim, D. Determination of the differential mode delay of a multimode fiber using Fourier-domain intermodal interference analysis. Opt. Express 14, 9016-9021 (2006).

Cheng, J. et al. Time-domain multimode dispersion measurement in a higher-order-mode fiber. Opt. Lett. 37, 347-349 (2012).

Xiao, Q., Yan, P., Ren, H., Chen, X. \& Gong, M. A side-pump coupler with refractive index valley configuration for fiber lasers and amplifiers. J. Lightwave Technol. 31, 3015-3022 (2013). Deng, L. The mnist database of handwritten digit images for machine learning research [best of the web]. IEEE Signal Process. Mag. 29, 141-142 (2012).

Borhani, N., Kakkava, E., Moser, C. \& Psaltis, D. Learning to see through multimode fibers. Optica 5, 960-966 (2018).

Li, Y. et al. Image Reconstruction Using Pre-Trained Autoencoder on Multimode Fiber Imaging System. IEEE Photonics Technol. Lett. 32, 779-782 (2020).

31 Cohen, G., Afshar, S., Tapson, J. \& Van Schaik, A. EMNIST: Extending MNIST to handwritten letters. 2017 International Joint Conference on Neural Networks (IJCNN). 2921-2926 (2017).

Xiao, H., Rasul, K. \& Vollgraf, R. Fashion-MNIST: a Novel Image Dataset for Benchmarking Machine Learning Algorithms. arXiv preprint, cs.LG/1708.07747 (2017).

Conner, J. L., Overlaur, M. \& Bhuva, R. L. Spatial light modulator with buried passive charge storage cell array. US patent 5,671,083 (1997).

34 Joshi, A. M., Heine, F. \& Feifel, T. Rad-hard ultrafast InGaAs photodiodes for space applications. Proc. SPIE 6220. 622003 (2006).

35 Atakaramians, S., Afshar, S., Monro, T. M. \& Abbott, D. Terahertz dielectric waveguides. $A d v$. Opt. Photonics 5, 169-215 (2013).

36 Velten, A. et al. Recovering three-dimensional shape around a corner using ultrafast time-offlight imaging. Nat. Commun. 3, 745 (2012).

37 Turpin, A., Musarra, G., Kapitany, V., Tonolini, F. \& Faccio, D. Spatial images from temporal data. Optica 7, 900-905 (2020).

Jalali, B., Jiang, Y. \& Karpf, S. Time stretch lidar: a fast spectrally scanned time-of-flight 3D camera. Proc. SPIE 11684. 116841B (2021).

39 Namihira, Y. ITU-T round robin measurement for nonlinear coefficient (n2/Aeff) of various single mode optical fibers. Technical Digest: Symposium on Optical Fiber Measurements, 2004. 33-36 (2004).

40 Keiser, G. Wiley encyclopedia of telecommunications (McGraw Hill, New York, 2000). 


\section{Supplementary Files}

This is a list of supplementary files associated with this preprint. Click to download.

- SupplementMaterial.pdf

- SupplementMaterial.pdf 